\title{
Phase Transition and Anisotropic Transport of MoS 2 /Chlorophyll Van der waal Heterostructure Formed via Biomimetic Photo-electron Donation
}

\section{Das}

Indian Institute of Technology Kharagpur

J. Sarkar Manna ( $\nabla$ jhimlimannaonline@gmail.com )

Indian Institute of Technology Kharagpur

T. K. Bhattaycharya

Indian Institute of Technology Kharagpur

\section{Research Article}

Keywords: Phase Transition, MoS2, Luttinger Transport, Van darwaalHeterostructure, Resonance tunneling, DFT, Negative differential resistance, chlorophyll

Posted Date: June 2nd, 2021

DOl: https://doi.org/10.21203/rs.3.rs-538884/v1

License: (c) (i) This work is licensed under a Creative Commons Attribution 4.0 International License. Read Full License 


\section{Abstract}

In atomically-thin two-dimensional Vander-Waal-heterostructure [VDWHs], phase transition due to biomimetic photoelectron donationwith molecular ad-layer has never been explored. In this pursuit, systematic quantification of biomimetic-optical-creation of stable easy-solution-processed 1T $\mathrm{MoS}_{2}$ chlorophyll (CHL-a) VDWHs has been examined. The 1T phase transformation dynamics and stabilization phenomenon have been quantified by optical anisotropy and Time-correlated-single-photoncounting. The material shows Luttinger transport phenomenon in the two-port device and supports $\mathrm{MoS}_{2}$ interfaces can be fine-tuned with the molecular ad-layer as a result of strong anisotropic finite range correlation. This is validated by Density-Function-Theory. The negative differential resistance in Luttinger transport arises from conformational heterogeneity of $\mathrm{CHL}$-a related to the scaling of Van der waal distances, which regulates coupling strength with temperature as supported by Molecular-Dynamics simulation. The photo-induced evolution of novel "anisotropic heterojunction" can stimulate a plethora of function-designable 2D VDWHs creation.

\section{Introduction}

The recent advancement in van der Waals (vdW) heterojunctions, two-dimensional (2D) Transition Metal Dichalcogenide (TMD) materials interface has created enormous opportunity for the fabrication of a wide range of electronic devices. By opting very simple colloidal synthesis method, (2D) TMD materials can be synthesized by low-cost and scalable manners, and its extended 2D surface can be functionalized with organic molecules, aiming towards specific electronic or optical applications like TFT, OLED, solar cell, sensors, etc. ${ }^{1,2,3,4}$. Unlike inorganic 2D Materials ,the molecular heterostructure with 2D counterpart offers the posiibility of unlimited degrees of freedom in molecular design, to introduce electronic, optical, and magnetic properties that can activate specific atomic/molecular interactions with 2D Materials. As large number of molecules collectively interacts with 2D Materials in those heterostructures macroscopic modulation in intrinsic properties becomes prominant instead of local variation introduced by single molecule. But the synthesis of stable 2D material molecular heterostructure is still in its infancy. Thus the idea of creating stable "molecular Van der Waal heterostructure" through an easy solution-processable route towards novel intrinsic macroscopic properties is worth executing ${ }^{5} . \mathrm{MoS}_{2}$ as an inorganic counterpart in molecular heterostructures has been successfully explored $3,6,7,6,7,8$. Though the formation of $2 \mathrm{H}$ molecular heterostructures is well documented, few data available in the literature showing $1 \mathrm{~T}$ $\mathrm{MoS}_{2}$ heterostructure with Porphyrin Molecular dopant.F4TCNQ has been added via physisorption with inert alkyl chains onto $\mathrm{MoS}_{2}$, in which parallel rows of dopant groups are separated by the interchains resulting in the alternating hole or electron-rich regions ${ }^{9}$. Recently Wann et. al. ${ }^{9}$ reported $1 \mathrm{~T}-\mathrm{MoS}_{2} / \mathrm{Ti}_{3} \mathrm{C}_{2}$ heterostructure by magneto-hydrothermal synthesis and the electrochemical storage mechanisms have been investigated. Similarly, Kwon et. al. reported a one-step hydrothermal reaction to $2 \mathrm{H}-1 \mathrm{~T}^{\prime}$ phase transition of $\mathrm{MoS}_{2}$ by intercalation of aromatic amine molecules ${ }^{10}$ via hydrothermal method through charge transfer mechanism with enhanced electrocatalytic activity ${ }^{11}$. Photoinduced phase transition 
without the requirement of temparature and pressure, in a simple solution processable method to produce $1 \mathrm{~T} \mathrm{MoS} 2$ heterostructure rarely been realised in literature. Not only that there are very few studies available that address the organic-inorganic hybrid system at the macroscopic level. A recent report by Gobbi et. al. has pointed out the possibilities of multiple potential wells when alkyl-chain conjugated molecules participate in heterostructure which can give rise to anisotropy in the electrical conduction of the twodimensional metal (2DM), though they were not aware of theoretical studies addressing the electronic or optical properties of such structures ${ }^{12}$. Finally Theoretical study of TMD and organic molecule heterostructure has been explored recently showing work function modulation in comparison with pure monolayer metal dichalcogenides ${ }^{13}$. But there is no theoretical study available to find out the extent and difference in anisotropic effective mass distribution between these van der waal molecular heterojunctions.

Here, we tried to shed light upon all of the lacunas.We propose one step evolution of a stable, solutionprocessable method for $1 \mathrm{~T} \mathrm{MoS}_{2}$ porphyrin-molecular heterostructure by photoconversion method, andintend to capture the macroscopic transport of $1 \mathrm{~T} \mathrm{MoS} 2 / \mathrm{CHL}$-a heterostructure in two-port devices. We also present details of theoritical analysis via DFT and molecular dynamics simulation to reasoning the macroscpic transport.

The choice of alkyl chain conjugated porphyrin's CHL-a molecules lies in the fact that it serves almost all of the criteria needed for exfoliation and electron donation. The $\mathrm{CHL}-\mathrm{a}$ amphiphilicity has been utilized to enhance the exfoliation of $2 \mathrm{H} \mathrm{MoS}_{2}$ in bi-solvent media to form a stable suspension of monolayer $\mathrm{MoS}_{2}{ }^{14}$. Das et. al. showed graphene $\mathrm{CHL}$-a nanohybrid 2D architecture where $\mathrm{CHL}$-a can act as a molecular wedge to produce graphene and the delocalized $\pi$-electrons over the stacked $\mathrm{CHL}$-a domain can contribute to the electron-transfer cascade of $2 \mathrm{D}$ graphene ${ }^{15}$. The photo-generated electrons of $\mathrm{CHL}-\mathrm{a}$ also have been utilized to reduce graphene oxide ${ }^{16}$ proving $\mathrm{CHL}$-a can be a molecule of choice to be successfully extended towards structural and functional modifications of other 2D materials.

This study can enable us to develop an efficient heterostructure with required functionalities by molecular design. The progression of the paper is as follows; we try to validate the time-dependent photoinduced formation of $1 \mathrm{~T} \mathrm{MoS}_{2}$ phase, [This has never been reported earlier in literature] with enhanced electron donation and electron-phonon coupling via TEM, Raman, and XPS data. Next analyzed the phase transformation dynamics with TCSPC and optical anisotropy data. We then quantify the $1 \mathrm{~T} \mathrm{MoS}$ phase formation dynamics with XPS, finally we validate the nonlinear macroscopic transport [Luttinger transport] phenomenon of $1 \mathrm{~T} \mathrm{MoS} / \mathrm{CHL}$-a heterostructure in a two-port device and substantiated this along with stabilization mechanism via density functional theory (DFT) and molecular dynamics (MD) study.

\section{Results And Discussion}


Raman signature Figure 1(a) represents the evolution of zone-center phonon $E_{2 g}^{1}$ and $A_{1 g}$ modes of the monolayer $2 \mathrm{H} \mathrm{MoS}_{2}$ [0 Minute photo exposure] with a wavenumber difference of $17.32 \mathrm{~cm}^{-1}$. An increase in the frequency of the $E_{2}^{1}$ gmode from the reported monolayer $2 \mathrm{H} \mathrm{MoS}_{2}$ is arising from either Coulomb interlayer forces or stacking induced changes in the intra-layer bonding via $\mathrm{CHL}$-a intercalation ${ }^{17,18}$. However while compared the Raman spectra of $2 \mathrm{H} \mathrm{MoS}_{2}{ }^{17}$ [Fig 1(a)] and CHL-a/2H MoS , we observed little broadening of $E_{2 g}^{1}$ modes which indicates nonuniform shear strain distribution, ${ }^{17,18}$ as nochange in $A_{1 g}$ mode is visible. Thus the presence of $\mathrm{CHL}$-a affects the equilibrium lattice parameter, resulting in the reduction of the lattice symmetry from $D 3 h$. This can lift the degeneracy of the $E_{2}^{1}{ }_{2}$ modes, hence the broadening and shifting of respective modes. This data also corroborates the observation of TEM showing lattice displacement of $0.04 \AA$ from pristine $2 \mathrm{H} \mathrm{MoS}_{2}(3.16 \AA)^{19}$ for $\mathrm{CHL}$-a exfoliated $\mathrm{MoS}_{2}$ [Fig. 1 (e)]. The presence of $\mathrm{CHL}-$ a can provide a significant substrate preventing random orientation, thus $2 \mathrm{H}$ $\mathrm{Mos}_{2}$ can be formed with $\mathrm{CHL}$-a assisted exfoliation [supporting information 1]. This has also been corroborated by XPS data in latersection ${ }^{20}$ where the shifting is maximized in CHL-a exfoliated sample.

Interestingly with increased light exposure, the $\mathrm{A}_{1 \mathrm{~g}}$ mode becomes sharp from exfoliated $2 \mathrm{H}$ counterpart, and $E_{2 g}^{1}$ line width increases [Fig. 1 (b)] implying enhanced electron-phonon coupling (EPC) in Photoexcited CHL-a with the occupation of the anti-bonding states.Electron doping leads to the occupation of the bottom of the conduction band of $\mathrm{MoS}_{2}$, [also further corroborated via DFT calculation] making the bonds weaker.The $A_{1 g}$ mode, which preserves the symmetry of the lattice, softens [Fig 1 (b)] because of the strengthening of electron-phonon coupling $\left(\lambda_{\mathrm{i}}\right)$ with doping. When the photo-exposure time is being elevated to 10 minutes the appearance of the reduced intensity of $E_{2 g}^{1}$ peak from that of 5 minutes photo-exposure found, and additional peaks, $156 \mathrm{~cm}^{-1}, 226 \mathrm{~cm}^{-1}$, and $330 \mathrm{~cm}^{-1}$ evolve, which can be explained in terms of the existence of a superlattice, as distorted octahedral structure with D3d symmetry ${ }^{17}$. The nature of the superlattice determines the particular point of the Brillouin-zone boundary that will be folded into the zone center and hence determines the phonons that will be observed after 10 minutes [the $156 \mathrm{~cm}^{-1}, 226 \mathrm{~cm}^{-1}$, and $330 \mathrm{~cm}^{-1}$ peaks of Fig [1 (a)]. These peaks correspond to frequencies at the $\mathrm{M}$ point of $\mathrm{MoS}_{2}{ }^{17}$ which appeared as a $2 \mathrm{a}_{0} \mathrm{Xa}_{0}$ superlattice in TEM. This distorted superlattice formation also evident from TEM and discussed in the following section with TEM. The formation of $1 \mathrm{~T}$ phase is observed, from a strong Raman band in 20 minutes photo exposure at $146 \mathrm{~cm}^{-1}$, attributed to Mo-Mo stretching vibrationsalong with. 219, 283, and $326 \mathrm{~cm}^{-1}$ supporting the TEM data ${ }^{18}$. We also found an $E_{2 g}^{1}$ peak, which is anomalous as no peak corresponding to trigonalbipyramidal symmetry of the $2 \mathrm{H}$ phase is expected to be found. From XPS it is evident that the conversion efficiency is $85 \%$ [Fig. $2(\mathrm{~m})$ ]remaining $2 \mathrm{H}$ phase could have contributed to this signal, but the intensity ratio, in that case, would have been the opposite. We found out that the $A_{1 g}$ peak intensity reduces to such an extent due to electron-phonon coupling after photo-exposure that a small $E_{2 g}^{1}$ peak corresponding to residual $2 \mathrm{H}$ peak becomes prominent. Due to the flattening of the $\mathrm{A}_{1 \mathrm{~g}}$ peak with electron donation discussed above, the $E_{2 g}^{1}$ peak intensity becomes prominent. As a result, the intensity ratio cannot determine the 
degree of phase transformation which has been evaluated as $85 \%$ from the XPS data in the later XPS section.

The EPC of the Raman mode at the Г-point of $\mathrm{MoS}_{2}$ exhibits a strong dependence on doping, similar to Kpoint phonons of graphene ${ }^{21}$. The electron-phonon coupling constant associated with a particular mode can be estimated from the Allen formula which relates the line width of the phonon mode to the dimensionless electron-phonon coupling constant ${ }^{21}$ :

\section{$\gamma_{i}=\frac{1}{g_{i}} \pi N\left(\epsilon_{f}\right) \lambda_{i} \omega_{b i}^{2}$}

where $N\left(\epsilon_{f}\right)$ is the electronic density of states at the Fermi level per eV per spin per unit cell (obtained from DFT calculation), $g_{i}$ is the mode degeneracy, with $g_{i}=1$ for an $A_{1 g}$ mode and $\omega_{b i}$ is the bare frequency in the absence of electron-phonon interaction. The full-width half maxima and frequency shift of two major bands $A_{1 g}$ and $E^{1}{ }_{2 g}$ is represented in [Fig.1 (c) and (d) ] respectively We estimated the scaling of the EPC parameter in 20 min photo-exposure to be $\lambda_{i}=6.084 \times 10^{-4}$ [calculation in supporting information 2] $]^{22}$ which justifies the strong coupling and the flattening of the $A_{1 g}$ peak.

The distortion in a lattice with photo exposure in 20 minutes can also be related to strain which helps in lower frequency shifting of $A_{1 g}$ mode. The presence of $\mathrm{CHL}-\mathrm{a}$ in the intercalated state with $6^{\text {th }}$ coordination as evident further in CHL-a Raman zone [supporting information 2; Fig.2s] can also induce strain $^{23}$.

In TEM Data we found diffraction pattern (EDP) with sharp Bragg spots [6 fold symmetry] alternating with dim ones in the (100) orientation under observation along the [010] zone-axis direction, which is following the $2 \mathrm{H} \mathrm{MoS}_{2}{ }^{24}$. A notable alteration of crystal symmetry is evident, after the light exposure. The $2 \times 2$ superstructure reflection appears along with the symmetry $(010)$ direction where the intensity of Bragg's spot becomesprominent[Fig. 1 (f)]. At 10 min photo exposer in Figure 1(g), the basic diffraction spots are in different degrees of brightness. The Bragg spots within green lines are more intense than yellow lines, indicating a phase transition from $2 \mathrm{H}$ to $1 \mathrm{~T} \mathrm{MoS}$. At 20 min photo exposer,[Fig. 1 (h)] the electron diffraction pattern moves from hexagonal $2 \times 2$ superstructure to orthogonal $2 x \sqrt{ } 3$ superstructure analogues to sodium intercalated $\mathrm{MoS}_{2}{ }^{24,25}$. This is due to enhanced electron transfer from CHL-a to $\mathrm{MoS}_{2}$ with time resulting in $2 x \sqrt{3}$ superstructure with lattice expansion. The electron transfer and probable Dynamics of photo conversion have been substantiated by TCSPC and optical anisotropy data and well supported by DFT calculation.[supporting Information 3\&4]

\subsection{Quantitative and qualitative evidence of phase conversion from XPS:}


The XPS spectra have been divided into three high-resolution regions corresponding to Mo $3 d, S 2 p$, and N1s to obtain a clear view of the 1T phase evolution and heterostructure formation. The binding energy of $\mathrm{Mo} 3 \mathrm{~d}$ in $2 \mathrm{H}_{-\mathrm{MoS}_{2}}$ [Fig. 2 (a)]features two principal peaks at around 229.5 and $232 \mathrm{eV}$ that correspond to $\mathrm{Mo}^{+} 3 \mathrm{~d} 5 / 2$ and Mo4${ }^{+} 3 \mathrm{~d} 3 / 2$ components, respectively for pristine $2 \mathrm{H} \mathrm{MoS}_{2} \cdot{ }^{26} \mathrm{After}$ photo exposure of 5 mint [Fig. 2 (b)]a new peak at $236.2 \mathrm{eV}$ and a deconvoluted peak at $233.0 \mathrm{eV}$ emerge in the XPS spectrum of $\mathrm{MoS}_{2} / \mathrm{CHL}-\mathrm{a}$, corresponding to $\mathrm{Mo6}^{+} 3 \mathrm{~d} 5 / 2$ and $3 \mathrm{~d} 3 / 2$ respectively which intensifies with photo- exposure time[Fig. 2(c)-(d)]. Down-shift of bonding energies also appears in the S 2p1/2 and $S$ $2 \mathrm{p} 3 / 2$ peaks as compared to doublet peaks of $2 \mathrm{H}-\mathrm{MoS}_{2}[\mathrm{~S} 2 \mathrm{p} 1 / 2$ at $163.2 \mathrm{eV}$ and S $2 \mathrm{p} 3 / 2$ at $162.2 \mathrm{eV}$ in the core-level S 2p] [Fig. 2(e)] with increased photo-conversion indicating the emergence of 1T phase ${ }^{20}$. [Fig. 2(f)-(h)]

In the $2 \mathrm{H}$ MoS2/CHL-a hybrid [without photo-exposure], Mo 3p peak at $395.2 \mathrm{eV}$ partially overlaps with N1S spectra of CHL-a, at a binding energy of 398.1-398.9 eV [Fig. 2(i)] which is characteristic of the pyrrolidine nitrogen atoms of the porphyrinmacro-cycle ${ }^{27}$. We found a second peak (N-2), a shoulder, at $400 \mathrm{eV}$ after photoexposure [Fig. 2 (j)], most likely due to the protonated nitrogen produced as a result of a small degree of de-metalization of $\mathrm{CHL}$-a during its exposure to X-rays in the course of the experiment. After gradual photo exposure[Fig. $2(\mathrm{k})-(\mathrm{l})$ ] peak N-3 gets intense at around $408.4 \mathrm{eV}$ with respect to 404 ev, indicating positively charged nitrogen of $\mathrm{CHL}-\mathrm{a}$ as a result of the photo-electron transfer, the expulsion of the core electrons from the $\mathrm{CHL}$-a nitrogen has become more difficult in this oxidized form thereby needing comparatively higher energy, i.e. $408.4 \mathrm{eV}$.in the photo- exposed CHL-a form. The positively charged nitrogen evolved is due to the transfer of non-bonding electrons on the nitrogen of $\mathrm{CHL}-\mathrm{a}$ to $\mathrm{MoS}_{2}$ after photo-exposure and $1 \mathrm{~T} \mathrm{MoS}{ }_{2}$ oxidized $\mathrm{CHL}-a$ gets stabilized. The presence of Mo6 ${ }^{+}$ ion in $1 \mathrm{~T}$ form also supports bandgap renormalization through Mo $4 \mathrm{~d}$ state as mentioned further in PDOS of DFT.

From XPS spectra the amount of conversion has been evaluated as $85.2 \%$ after 20 minutes of photo exposure[Fig. $2(\mathrm{~m})]^{27}$ by peak area contributions of $1 \mathrm{~T} \mathrm{~S} 2 \mathrm{p} 1 / 2$ and $S 2 \mathrm{p} 3 / 2$ peaks in S $2 \mathrm{p}$ highresolution region of $\mathrm{MoS}_{2}$.

\subsection{Transport properties:}

To evaluate the macroscopic transport phenomenon along with the applicability of this material in device conformation wesubjected this material via simple drop-casting to construct two-terminal device architecture [details of device fabrication is in supporting information 5]. The $1 \mathrm{~T} \mathrm{MoS} / \mathrm{CHL}$-a currentvoltage curve is non-ohmic [Fig 3 (a)]. Another interesting phenomenon that is observed here is the temperature-dependent negative differential resistance (NDR) effect [Fig 3 (a)]. The current-voltage characteristics have been discussed in detail in the following section. The NDR effect has been evaluated in the later section by correlating MD simulation. 


\subsubsection{Current-voltage characteristics:}

Considering the power-law dependence [IV $\left.{ }^{\beta+1}\right]$ of current-voltage characteristics, we correspond this transport to non-Fermi liquid or Luttinger liquid behavior. Angle-integrated studies ${ }^{28}$ of quasi-2D organic metals have always reported a power-law dependence of the density of states, suggestive of Luttinger liquid behavior like in the quasi-1D organic metals ${ }^{29}$. The $T$ and $V$ dependence for tunneling into a 1D LuttingerLiquidvia Fermi-liquid metal contacts is given by

$$
I=J_{0} T^{1+\alpha} \sinh \left(\frac{\gamma e V}{2 k T}\right)\left|\Gamma\left(1+\frac{\beta}{2}+i \frac{\gamma e V}{2 \pi k T}\right)\right|^{2}
$$

Where $a=\left(g^{-1}-1\right) / 4, \beta=\left(g+g^{-1}-2\right) / 8 J_{0}$ is a constant, and the Luttinger parameter $g=\vartheta_{F} / \vartheta_{\rho}$ is a fitting parameter that accounts for the voltage drop over the circuit $^{30}$.

To validate thisLuttinger behavior a collapse diagram of the transport characteristic is obtained by plotting $\mathrm{l} / \mathrm{T}^{\mathrm{a}+1}$ against eV/kT. Where $\mathrm{a}$ is the slop of zero-voltage conductivity against temperature [Fig 3 (b)]. We found the $a=7.26$ (and $\gamma^{-1} \approx 1000, \beta=12.007$ ); Plotting the entire data set $/ / T^{a+1}$ against $\mathrm{eV} / \mathrm{kT}$ according to the Luttinger Liquid prediction. In our case, the data collapse quite well onto a single curve confirming Luttinger LiquidFig. 3 (b) ${ }^{31}$.

\subsection{Mechanism of anisotropic transport via DFT:}

- The two-dimensional (2D), highly dispersive interface states of $\pi$-conjugated organic molecules and a metal surface have been described theoretically and experimentally as strongly dispersive anisotropic, introducing some effective 1D potential ${ }^{32}$. Analogous to this, we tried to investigate the underlying mechanism of Luttinger transport with DFT calculation.

Our DFT calculations indicate several important differences between bare 1T $\mathrm{MoS}_{2}$ and $1 \mathrm{~T} \mathrm{MoS}_{2} / \mathrm{CHL}-\mathrm{a}$ [Fig. 3 (e) \& (f)]. Compared to shallow electron pocket in bare 1T MoS $2,1 \mathrm{~T} \mathrm{MoS} / 2$ CHL-shows a deep electron pocket at $\Gamma$ high symmetry point. The anisotropic dispersion of band on either direction of $\Gamma$ point is evident which arises from the cross over at point-(ii)[ Fig. 3 (e), (f)] towards $\Gamma$-M direction and dispersion towards $\Gamma-k$ direction at the point-(ii). In bare $1 \mathrm{~T} \mathrm{MoS}_{2}$ the highly dispersive band arising from a spin-orbit coupling on either side of $\Gamma$ forming a small electronic pocket at $-250 \mathrm{mv}$ via large energy splitting $^{33}$ [Fig. 3 (e) point-(ii)] in small momentum space while compared to $1 \mathrm{~T} \mathrm{MoS}_{2}$. This indicates spinorbit interaction and lesser inter-orbital interaction in $2 \mathrm{H} \mathrm{MoS}_{2}$ since orbitals are deformed by the atomic bonding. On the flip side, $1 \mathrm{~T} \mathrm{MoS}_{2} / \mathrm{CHL}$-a shows lesser dispersion and band crossing in larger 
momentum space [complying strong interorbital interaction on either side of symmetry points $\Gamma$ [Fig. 3 (e)]. PDOS around Fermi level [Fig. 3 (c), (d)] points out that this evolved through the s3p and Mo 4d orbital with lifted degeneracy in DOS at "zero" bias [Fig. 3 (c), (d)]. Resulting in type-ll Dirac points like dispersion and electronic pocket indicating strong finite range correlation [Fig 3 (f)point-(ii)]. This type of band which is not prominent in bare counterpart generally evolved with a topologically ordered state. Though it calls for further experimental verification this theoretical input gives the first glimpse of the probable existence of the exotic state in $1 \mathrm{~T} \mathrm{MoS}_{2} / \mathrm{CHL}-\mathrm{a}$ VDWH. Doping induced via $\mathrm{CHL}$-a interaction, as verified via the experimental section enhances the energy value of the electronic pocket around $\Gamma$ point up to -400 mev specifically indicating strong finite range correlation. Another interesting feature arises around $\Gamma$ point $1 \mathrm{mev}$ away from Fermi level. The two-fold band degeneracy in bare $1 \mathrm{~T} \mathrm{MoS}_{2}$ at $1 \mathrm{mev}$ from $\Gamma$ to $\mathrm{M}$ and $\Gamma$ to $\mathrm{K}$ points is lifted in $1 \mathrm{~T} \mathrm{MoS}_{2} / \mathrm{CHL}$-a and a new set of orbital dispersion combination evolve via splitting and band lift off around 300 mev with strong asymmetry at $\Gamma$ point which indicates strong spin-orbital coupling interaction ${ }^{34}$, this results in new inter-band asymmetry formation at 600 mev closer to the $499 \mathrm{mev}$ minima of electron pocket, opening a gap probably showing directionality for quasiparticle movement [Fig. 3 (f) point-(ii)]. Thus at $\Gamma$ symmetry point highly dispersive band with enhanced spin-orbit coupling is prominent in the $1 \mathrm{~T} \mathrm{MoS} / \mathrm{CHL}$-a system. This entire feature especially electronic pocket signifying quantization of carrier is one of the signatures of the Luttinger Liquid phenomenon we observed here. The 1D nature can be further illustrated via anisotropic effective mass distribution [Fig. 3 (g) (h) $]^{35}$. The calculated effective mass of $1 \mathrm{~T} \mathrm{MoS}_{2} / \mathrm{CHL}$-a [for details, Supporting information 5] shows, charge carriers are in lower order that is $0.58 \mathrm{X} \mathrm{m}_{0}$ (where $\mathrm{m}_{0}=$ free electron effective mass) for the electrons moving in the $\Gamma$ to $\mathrm{M}$ direction than $\Gamma$ to $\mathrm{K}$ direction $\left(1.32 \mathrm{X} \mathrm{m}_{0}\right)$ corresponding to highly dispersive and flat curve in the $\mathrm{K}$ space respectively. Lower dispersion could be attributed to the different confinement of quasi 1D structure while flattening indicates localization. The band flattening indicated by the a parameter depicting nonparabolicity is higher in $\Gamma$ to $\mathrm{K}$ direction due to increased transport effective mass. The localization may have occurred due to the proximity of $\mathrm{CHL}-\mathrm{a}$ r-electrons forming Van der waal interaction which also creates lattice changes in the supercell. The band edge dispersion states strong anisotropy with a value as 0.30434 and 1.00706 while compared to bare counterpart showing highly dispersive confinement of quasiparticle.

A clear visual of the finite contribution of each of the s3p and $4 \mathrm{~d}$ orbital [Fig. 3 (d)] is evident while in bare $1 \mathrm{~T} \mathrm{MoS}$ the s3p and Mo 4d orbital contribute the degenerative higher value of density of state [Fig. 3 (c)]. The lifting of degeneracy in DOS around "zero" bias in $1 \mathrm{~T} \mathrm{MoS} / 2 \mathrm{CHL}$-a gives rise to asymmetric effective mass distribution corresponding to the shape of the orbital emphasizing directionality. The particle-hole asymmetry is prominent in $1 \mathrm{~T} \mathrm{MoS}_{2} / \mathrm{CHL}$-a. The asymmetry of the density of states is caused by the nontrivial interplay of the spin and charge degrees of freedom. The pseudo-gap-like structure shed light on the possibility of the directional movement of electrons. This can be related to the lift-off orbital degeneracy near the electron pocket above the Fermi level opening a gap at the Brillouin zone boundary which is evident in $1 \mathrm{~T} \mathrm{MoS}_{2} / \mathrm{CHL}$-a hole pocket [Fig. 3 (e), (f) (iii)]. ${ }^{36}$. 


\subsection{Resonance tunneling (RT) in Luttinger liquid via Van der waal screening:}

Another important feature we can find in the IV curve is the Negative differential resistance which gradually becomes diffusive with lower temperature. Considering the VDWH is a molecular interface we explain this owing to conformational heterogeneity of $\mathrm{CHL}$-a to different temperatures as evident from MD simulation. Resonance tunneling occurs with the level alignment of CHL-a molecular orbital with that of $1 \mathrm{~T} \mathrm{MoS}$. In higher temperature $323 \mathrm{~K}$, we observed the onset energy is little negative or very close to zero with a sharp resonance peak, implying that tunneling direction is from 1T MoS 2 to CHL-a [considering $\mathrm{CHL}-\mathrm{a}$ intercalated within $\mathrm{MoS}_{2}$ layer]. As the temperature reduces [from $323 \mathrm{~K}$ to $293 \mathrm{~K}$ ] the onset energy gradually shifts to positive energy with diffusive NDR peaks. The shifts may be correlated to the directional flip of electron transfer, that is electron is now tunneled from CHL-a to $1 \mathrm{~T} \mathrm{MoS}$ where higher energy is needed to bring the molecular orbital resonantly accessible to each other in the hightemperature range [Fig. 4 (a) $]^{14}$. This is because the conformation of $\mathrm{CHL}-\mathrm{a}$ at this ascending temperature range gradually becomes side by side attractive as evident from Fig. 4 (b) and (c). Now we correlate this phenomenon with the Luttinger Liquid resonance tunneling theory. [supplementary 4\&5]

And found out [ Fig. 4 (d)] the reduction of repulsive scattering[g] as transmission probability $\left(\Gamma_{i}\right)$ enhanced with temperature stemming from the scaling of Van der waal distance between CHL-a and 1T $\mathrm{MoS}_{2}$. This is preserved upon CHL-a conformational heterogeneity. At a temperature above $293 \mathrm{~K}$ the system shows NDR Fig. 4 (f)-(i) and below, the system conductance becomes linear [supporting information 6, Fig. 6S].

As the temperature increases the Van der waal distance also increases, but Van der waal energy of the whole system first decreases then increases, and finally goes down to a minimum [Fig. 4 (b)]. This is because our proposed system can act as an electrostatic double barrier configuration of polarizable CHLa and $1 \mathrm{~T} \mathrm{MoS}$, hence system potential energy is predominated by effective interaction of Van der waal attraction energy which is caused by the formation of induced dipoles between polarizable materials which are much stronger on a short distance [between 4.5 to $9.5 \AA$ ]. On the other hand polarizability of $\mathrm{CHL}-\mathrm{a}$, in turn, depends upon the molecular orientation with refference to $1 \mathrm{~T} \mathrm{MoS}_{2}$ plane. Head-to-tail interaction [molecular Z-axis perpendicular to xy-plane of $\mathrm{MoS}_{2}$ ] is attractive and side-by-side interaction [the deviation of Z-axis from perpendicular position xy-plane] becomes repulsive ${ }^{37}$. Starting from $263 \mathrm{~K}$ as the distance increases with temperature, the configuration gets tilted and changes to head to tail [Fig. 4 (b) and (c)], hence energy is minimized and a local minimum is formed at temperature 273k. Further enhancement of temperature increases the distance, molecular orientation changes to the side by side repulsive configuration, and energy increases [283K to $293 \mathrm{~K}$ ]. It attains the maxima when torsion energy is $35.5 \mathrm{kcal} / \mathrm{mol}$ and temperature is $293 \mathrm{k}$. After $293 \mathrm{~K}$ the orientation becomes head to tail, with gradual reduction of Van der waal energy, finally finding a second minimum at temperature $323 \mathrm{~K}$ with reduced 
torsional energy $31 \mathrm{kcal} / \mathrm{mol}$ [Fig. 4 (e)]. This energy minimization is caused by the almost perpendicular molecular z-axis orientation of CHL-a with $1 \mathrm{~T} \mathrm{MoS}_{2}$ on the xy-MoS${ }_{2}$ plane.

The cross-over point where the Van der waal distance increases with temperature and the Van der waal energy of the system decreases is around 300k. Experimentally around this point (crossover point), we started observing the NDR effect.

The NDR effect as well as the transmission probability directly related to Van der waal distance and the level alignment of the molecular orbital. Only the level alignment depends upon configuration which changes upon temperature resulting in a temperature-dependent NDR effect. Hence at a temperature above $293 \mathrm{~K}$, the configuration is such that Van der waal energy minimizes, but at the same time distance and transmission probability increased and level alignment maximizes, cumulatively giving rise to a stable structure having an NDR effect in high temperature. We observed from the simulation that the system became stabilized at temperatures 263K [point A] and 323K[point B].;

Though we find the same energy regime in low distance without NDR peak because Van der waal screening is higher due to hybridization in the lower distance with no tunneling probability and the molecules enter into the off-resonance situation. At a higher distance, the coupling between CHL-a and $\mathrm{MoS}_{2}$ reduces which in turn minimizes the screening effect and the system enters into a highly resonating regime ${ }^{38}$. Thus this material can act in two energy regimes where energy is minimized first in low temperature where the Luttinger phenomenon is prominent and second high temperature where the NDR effect is profound [Fig. 4 (a) scheme].

\section{Conclusion}

We have successfully synthesized phase selective $1 \mathrm{~T} \mathrm{MoS}_{2} / \mathrm{CHL}$-a heterointerface by combining exfoliation and photoelectron donation process with the help of a unique biomolecule "Chlorophyll" having both amphiphilicity and electron-donating properties. The gradual phase transformation from $2 \mathrm{H}$ to $1 \mathrm{~T}$ is evident from Raman and TEM data, revealing increased electron-phonon couplings augmented with alteration of crystal symmetry. This also substantiated biexponentially fitted TCSPC curve in the selfassembled $\mathrm{CHL}-\mathrm{a}$ emission region where the lifetime is very fast indicative of non-radiative pathway. Through optical anisotropy, we tried to explain the heterostructure formation dynamics in the following manner; At first electronically coupled CHL-a units, participating in $\mathrm{MoS}_{2}$ phase conversion, increases quantitatively with time. The rotational degree of freedom of these units also reduces with time and more ordered structures are formed over the $\mathrm{MoS}_{2}$ surface. Modulation of electronic structure mainly happens in Mo $4 \mathrm{~d}$ and $4 \mathrm{~s}$ orbital state as evident from XPS confirming around $85 \%$ conversion efficiency within the experimental time window. The Luttinger transport property in a two-port device has been substantiated theoretically via DFT calculation. The band edge dispersion around the high symmetry point is prominent in theoretical observation, which states strong electronic anisotropy, confirming the 
highly dispersive confinement of quasi-particles. Lifting of the degeneracy of PDOS around zero bias of s3p and Mo $4 \mathrm{~d}$ orbital in $1 \mathrm{~T} \mathrm{MoS}_{2} / \mathrm{CHL}$-a from bare counterpart clearly states the directionality of electron transport. The asymmetry of the density of states is caused by the nontrivial interplay of the spin and charge degrees of freedom in the $1 \mathrm{~T} \mathrm{MoS} / \mathrm{CHL}$-a heterostructure with anisotropic effective mass distribution. The high-temperature NDR diffuses off as the temperature decreases as in the case of resonance tunneling of Luttinger liquid. This can be correlated with temperature-dependent conformational heterogeneity of CHL-a over the $1 \mathrm{~T}$ surface. Depending upon the $\mathrm{z}$ molecular axis over $\mathrm{XY}$-plane the conformation becomes repulsive (side by side) and attractive (head to tail) resulting in two minima. The system shows only Luttinger transport in 1st minima (273K) and NDR in Luttinger transport 2nd minima (323K). Interestingly we found a crossover temperature (300K) that separates the whole transport region into two specific regimes. Below $300 \mathrm{~K}$ temperature, there is only a Luttinger transport region, where the hybridization increases and no tunneling present. Above the crossover temperature, the NDR-dominated region, where the tunneling probability arises with a gradual increase in distance. In conclusion, the experimental findings supported via the theoretical framework of 2DMs with molecular systems represent a viable protocol to develop multifunctional hybrid materials and devices suitable for advanced logic, memory, and sensing applications. Keeping in view the features like type-II Dirac cone, pseudogap, band crossing at larger momentum space in $1 \mathrm{~T} \mathrm{MoS} / \mathrm{CHL}-a$ evident from DFT calls for further experimental evidence to evaluate any room temperature topologically protected structure in the system.

\section{Experimental Section/ Methods}

We inserted chlorophyll-a molecules into the $\mathrm{MoS}_{2}$ through exfoliation in water-alcohol media, similar to the process Das et. al. opted for grapheme exfoliation ${ }^{14}$. The water content of $10 \mathrm{~mL}$ of $\mathrm{MoS}_{2}[1 \mathrm{mg} / \mathrm{mL}]$ in ethanol (SigmaAldrich, ACS reagent, $>99.5 \%$ ) solution, gradually increased along with low power sonication $(120 \mathrm{~W})$, so that the amphiphilic $\mathrm{CHL}$-a molecules [conc $10^{-7} \mathrm{~mol}$ ], present in alcohol, enter into $\mathrm{MoS}_{2}$ by finding their way to the gaps opening up at the edges through sonication to minimize hydrophobic interaction causing a substantial volume expansion of the $\mathrm{MoS}_{2}$ crystal, where interlayer spacing increases from the original $3.16 \AA$ to $3.2 \AA$. The dimension of molecules may be used to tailor the structure (such as interlayer spacing and molecule packing density) and properties (electron-doping level) of intercalatedcompounds. The resultant nanosheets were washed repeatedly through centrifugation, 2,000 r.p.m. for $3 \mathrm{~min}$ to remove the excess CHL-a and large aggregates, and then dispersed in DI water to formulate a stable and easy-to-handle $\mathrm{MoS}_{2}$ solution. The slightly greenish-yellow color [Figure 5S (a)\& (b)] of the dispersion is indicative of the formation of relatively thin, semiconducting nanosheets.

\section{Declarations}

\section{Author Contributions}


D. Das acquired and analyzed data, contributed to designing the project, and did the theoretical calculation. J Manna conceptualized and designed the project, analyzed the experimental and theoretical data, and wrote the manuscript. T. K. Bhattacharyyahelped in experiments and data acquisition. All authors read and approved the final manuscript.

\section{Acknowledgements}

Financial support from the Department of Science and Technology, India under the Woman Scientist Scheme (WOS-A; Project Reference No. SR/WOS-A/ET-15/2017) to Dr. Jhimli Sarkar Manna is gratefully acknowledged.

\section{References}

1. Yu, W. J. et al. Highly efficient gate-tunable photocurrent generation in vertical heterostructures of layered materials. Nat. Nanotechnol.8, 952-958 (2013).

2. Furchi, M. M., Pospischil, A., Libisch, F., Burgdörfer, J. \& Mueller, T. Photovoltaic effect in an electrically tunable Van der Waals heterojunction. Nano Lett.14, 4785-4791 (2014).

3. Park, S. Y. et al. Room temperature humidity sensors based on $\mathrm{rGO} / \mathrm{MoS} 2$ hybrid composites synthesized by hydrothermal method. Sensors Actuators, B Chem.258, 775-782 (2018).

4. Sekitani, T. et al. Stretchable active-matrix organic light-emitting diode display using printable elastic conductors. Nat. Mater.8, 494-499 (2009).

5. Oyedele, A. D., Rouleau, C. M., Geohegan, D. B. \& Xiao, K. The growth and assembly of organic molecules and inorganic 2D materials on graphene for van der Waals heterostructures. Carbon $\mathrm{N}$. Y.131, 246-257 (2018).

6. Liu, F. et al. Van der Waals p-n Junction Based on an Organic-Inorganic Heterostructure. Adv. Funct. Mater.25, 5865-5871 (2015).

7. Vélez, S. et al. Gate-tunable diode and photovoltaic effect in an organic-2D layered material p-n junction. Nanoscale7, 15442-15449 (2015).

8. Jariwala, D. et al. Hybrid, Gate-Tunable, van der Waals p-n Heterojunctions from Pentacene and MoS2. Nano Lett.16, 497-503 (2016).

9. Wang, X. et al. 2D/2D 1T-MoS2/Ti3C2 MXene Heterostructure with Excellent Supercapacitor Performance. Adv. Funct. Mater.30, 1-11 (2020).

10. Kwon, I. S. et al. Intercalation of aromatic amine for the $2 \mathrm{H}-1 \mathrm{~T}$ ' phase transition of MoS2 by experiments and calculations. Nanoscale10, 11349-11356 (2018).

11. Kwon, I. S. et al. Two dimensional MoS2 meets porphyrins: Via intercalation to enhance the electrocatalytic activity toward hydrogen evolution. Nanoscale11, 3916-3924 (2019).

12. Gobbi, M. et al. Periodic potentials in hybrid van der Waals heterostructures formed by supramolecular lattices on graphene. Nat. Commun.8,1-8 (2017). 
13. Osman, M. A., Rashid, M. M., Aziz, M. A., Habib, M. R. \&karim, M. R. Inhibition of Ehrlich ascites carcinoma by Manilkarazapota L. stem bark in Swiss albino mice. Asian Pac. J. Trop. Biomed.1, 448-451 (2011).

14. Das, D., Sarkar Manna, J. \& Mitra, M. K. Electron donating chlorophyll a on graphene: A way toward tuning fermi velocity in an extended molecular framework of graphene/chlorophyll a nanohybrid. J. Phys. Chem. C119, 6939-6946 (2015).

15. Das, D., Sarkar Manna, J. \& Mitra, M. K. Electron Donating Chlorophyll-a on Graphene: A Way toward Tuning Fermi Velocity in an Extended Molecular Framework of Graphene/Chlorophyll-a Nanohybrid. J. Phys. Chem. C119, 6939-6946 (2015).

16. Das, D., Sarkar Manna, J. \& Mitra, M. K. Unravelling the photo-excited chlorophyll-a assisted deoxygenation of graphene oxide: Formation of a nanohybrid for oxygen reduction. RSC Adv.5, 65487-65495 (2015).

17. Zhou, K. G. et al. Raman Modes of MoS2 Used as Fingerprint of van derWaals Interactions in 2-D Crystal-Based Heterostructures. ACS Nano8, 9914-9924 (2014).

18. Liang, L. et al. Low-Frequency Shear and Layer-Breathing Modes in Raman Scattering of TwoDimensional Materials. ACS Nano11, 11777-11802 (2017).

19. Yan, A. et al. Dynamics of Symmetry-Breaking Stacking Boundaries in Bilayer MoS2. J. Phys. Chem. C121, 22559-22566 (2017).

20. Sim, D. M. et al. Controlled Doping of Vacancy-Containing Few-Layer MoS2 via Highly Stable ThiolBased Molecular Chemisorption. ACS Nano9, 12115-12123 (2015).

21. Schafer, K. J. et al. Superconductivity in the Fullerenes. 3999, 989-992 (1991).

22. Wu, S. F. et al. Raman scattering investigation of the electron-phonon coupling in superconducting $\mathrm{Nd}(\mathrm{O}, \mathrm{F})$ BiS2. Phys. Rev. B - Condens. Matter Mater. Phys.90, 1-5 (2014).

23. Harivyasi, S. S., Hofmann, O. T., Ilyas, N., Monti, O. L. A. \& Zojer, E. Van der Waals Interaction Activated Strong Electronic Coupling at the Interface between Chloro Boron-Subphthalocyanine and Cu(111). J. Phys. Chem. C122, 14621-14630 (2018).

24. Wang, L., Xu, Z., Wang, W. \& Bai, X. Atomic mechanism of dynamic electrochemical lithiation processes of MoS2 nanosheets. J. Am. Chem. Soc.136, 6693-6697 (2014).

25. Heising, J. \& Kanatzidis, M. G. Structure of restacked MoS2 and WS2 elucidated by electron crystallography. J. Am. Chem. Soc.121, 638-643 (1999).

26. Leng, K. et al. Phase Restructuring in Transition Metal Dichalcogenides for Highly Stable Energy Storage. ACS Nano10, 9208-9215 (2016).

27. Das, D., Sarkar Manna, J. \& Mitra, M. K. Unravelling the photo-excited chlorophyll-a assisted deoxygenation of graphene oxide: formation of a nanohybrid for oxygen reduction. RSC Adv.5, 65487-65495 (2015).

28. Xue, M. et al. Room temperature negative differential resistance of a monolayer molecular rotor device. Appl. Phys. Lett.95, 2-5 (2009). 
29. Jezouin, S. et al. Tomonaga-Luttinger physics in electronic quantum circuits. Nat. Commun.4, (2013).

30. Bockrath, M. et al. Luttinger-liquid behaviour in carbon nanotubes. Nature397, 598-607 (1999).

31. Uplaznik, M., Bercic, B., Remskar, M. \& Mihailovic, D. Quantum charge transport in Mo6 S3 I6 molecular wire circuits. Phys. Rev. B - Condens. Matter Mater. Phys.80, 1-6 (2009).

32. Hacker, C. A. \& Hamers, R. J. Optical and electronic anisotropy of $\pi$-conjugated molecular monolayer on the silicon(001) surface. J. Phys. Chem. B107, 7689-7695 (2003).

33. Keum, D. H. et al. Bandgap opening in few-layered monoclinic MoTe 2. Nat. Phys.11, 482-486 (2015).

34. Goodenough, J. B. Spin-orbit-coupling effects in transition-metal compounds. Phys. Rev.171, 466479 (1968).

35. Stühler, R. et al. Tomonaga-Luttinger liquid in the edge channels of a quantum spin Hall insulator. Nat. Phys.16, 47-51 (2020).

36. Yuen, J. D. et al. Nonlinear transport in semiconducting polymers at high carrier densities. Nat. Mater.8, 572-575 (2009).

37. Bergmann, K. et al. Roadmap on STIRAP applications. J. Phys. B At. Mol. Opt. Phys.52, 202001 (2019).

38. Fereiro, J. A. et al. Tunneling explains efficient electron transport via protein junctions. Proc. Natl. Acad. Sci. U. S. A.115, E4577-E4583 (2018).

\section{Figures}
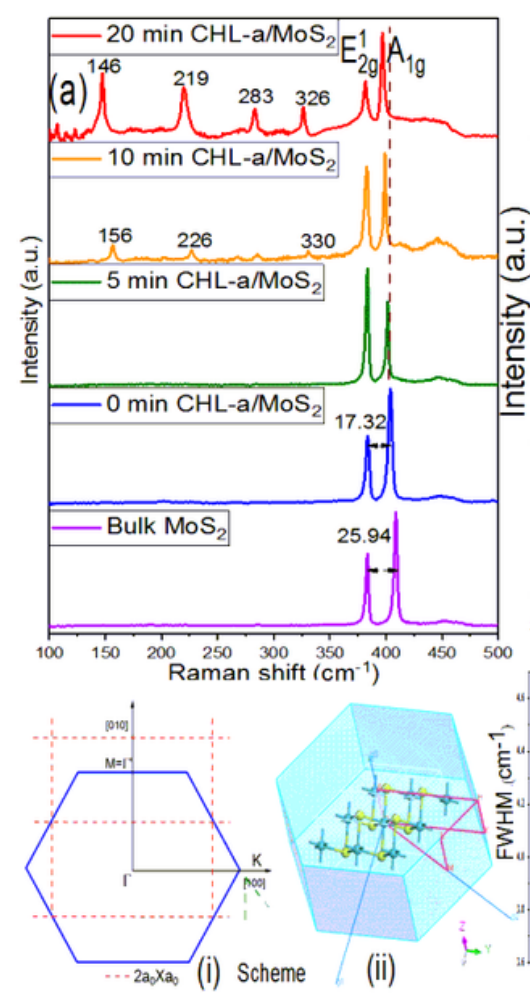
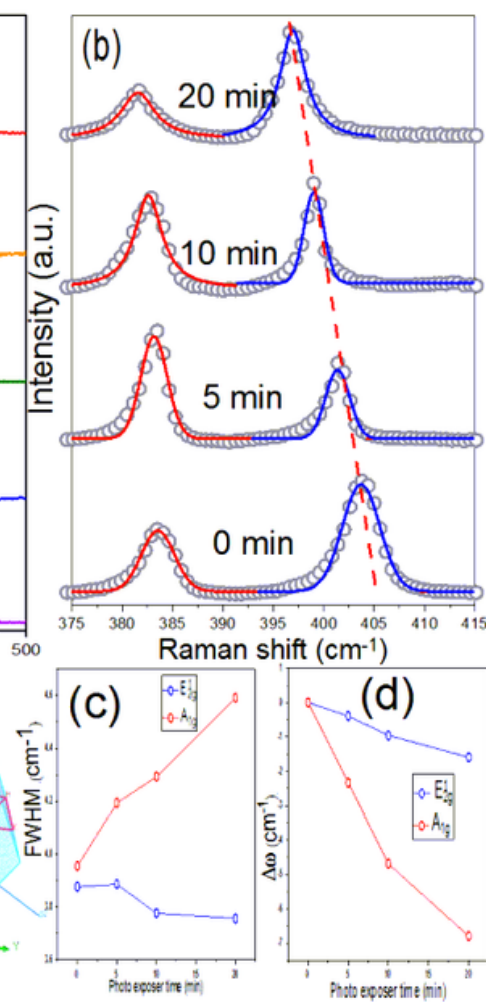

(d)

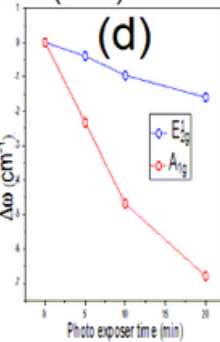

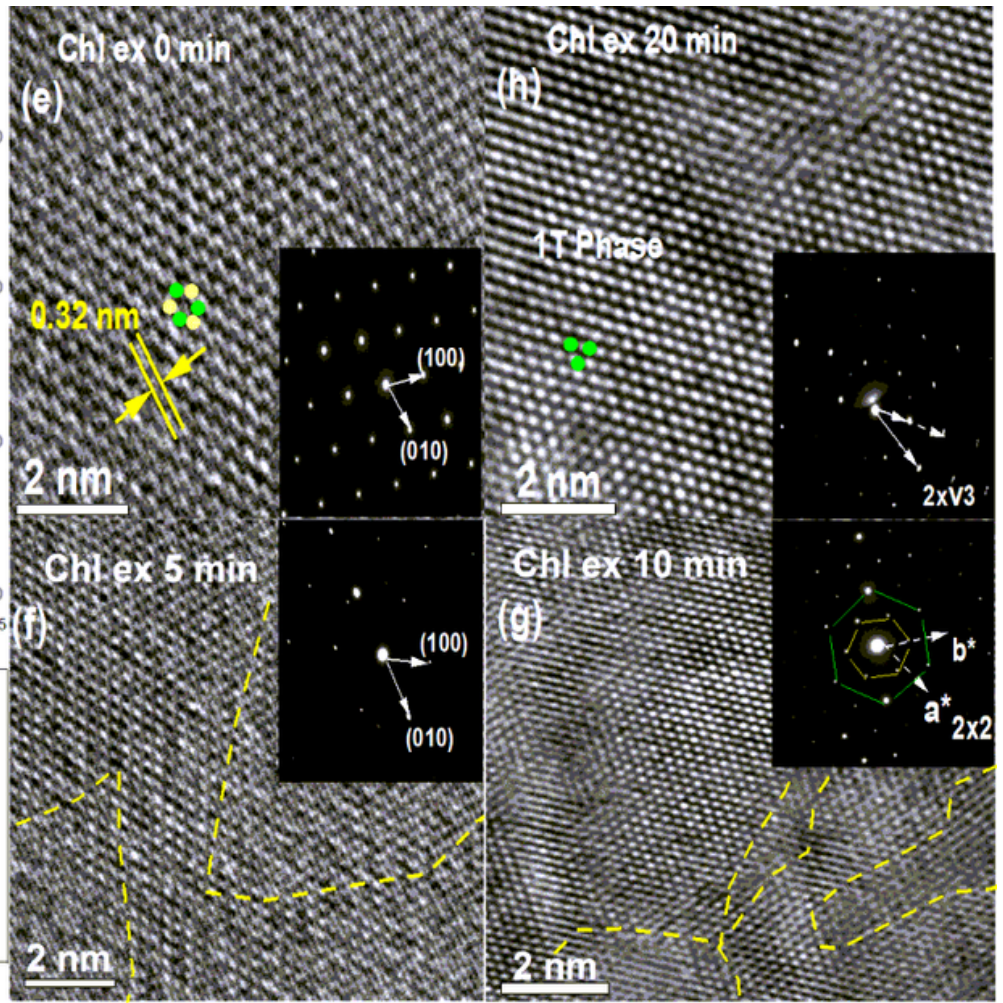


Raman spectra of as-exfoliated and photo-exposed MoS2 with time [a]. Shifting of A1g peak with photo exposure is visible from [b] depicting phase transformation. An estimation of full-width half maxima and frequency shift of two major bands A1g and E12g is represented in [c] and [d] respectively. Scheme: (i) \& (ii) Reciprocal space diagram illustrating the relationship between the basal plane projection of the Brillouin zone of 2H MoS2 (solid lines) and two-dimensional Brillouin zone of a 2a0Xa0 superlattice. [e]TEM images of $2 \mathrm{H}$ MoS2 honeycomb lattice with an Electron Diffraction Pattern (EDP) of sharp Bragg spots [ 6 fold symmetry]. Gradual photo exposure leads to notable alteration of crystal symmetry along with intermediate superstructure formation as evident from lattice and Selected Area Electron Diffraction (SAED) [f,g] focusing light on phase transformation dynamics. Finally, 1T phase with hexagonal lattice is evident[h]. The yellow dashed line signifies the zone boundary of 2H and 1T MoS2 lattice.
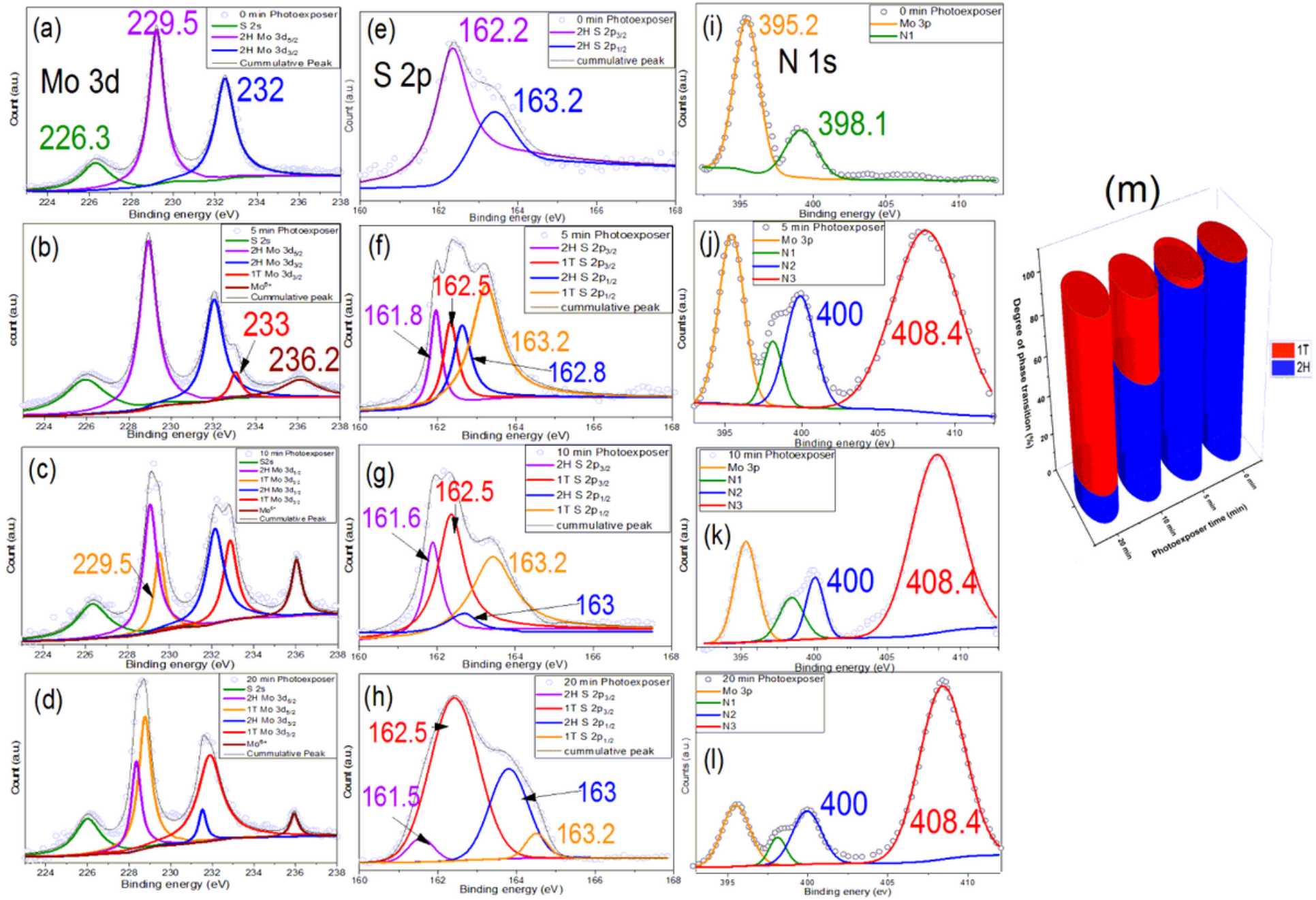

\section{Figure 2}

XPS spectra of Mo 3d [a,b,c,d] s2p [e,f,g,h] showing gradual evolution of 1T phase after photoexposure.N1s (i,j,k,l) XPS spectra show positively charged nitrogen evolved due to the transfer of non-bonding electrons on the nitrogen of CHL-a to MoS2 after photo-exposure. $(\mathrm{m})$ Degree of phase transition from $2 \mathrm{H}$ MoS2 to 1T MoS2 under time-dependent photo exposer. 

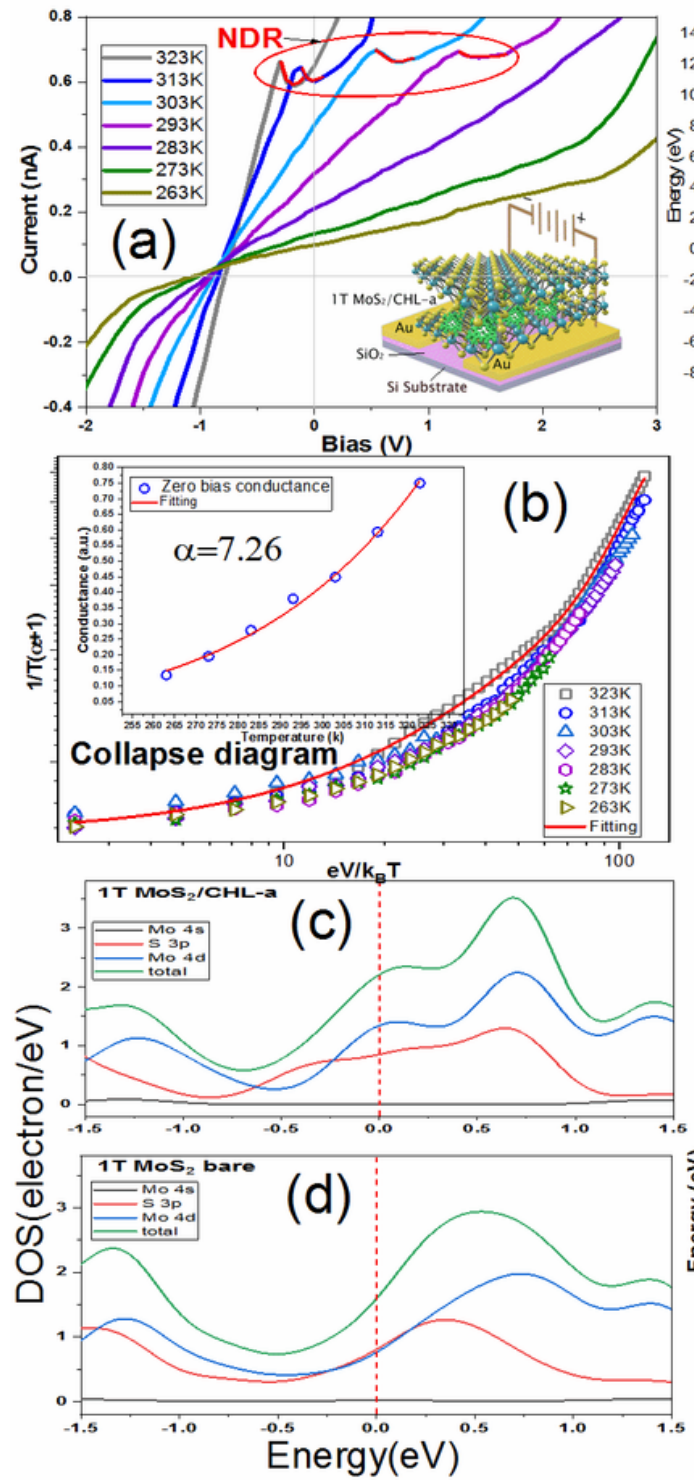
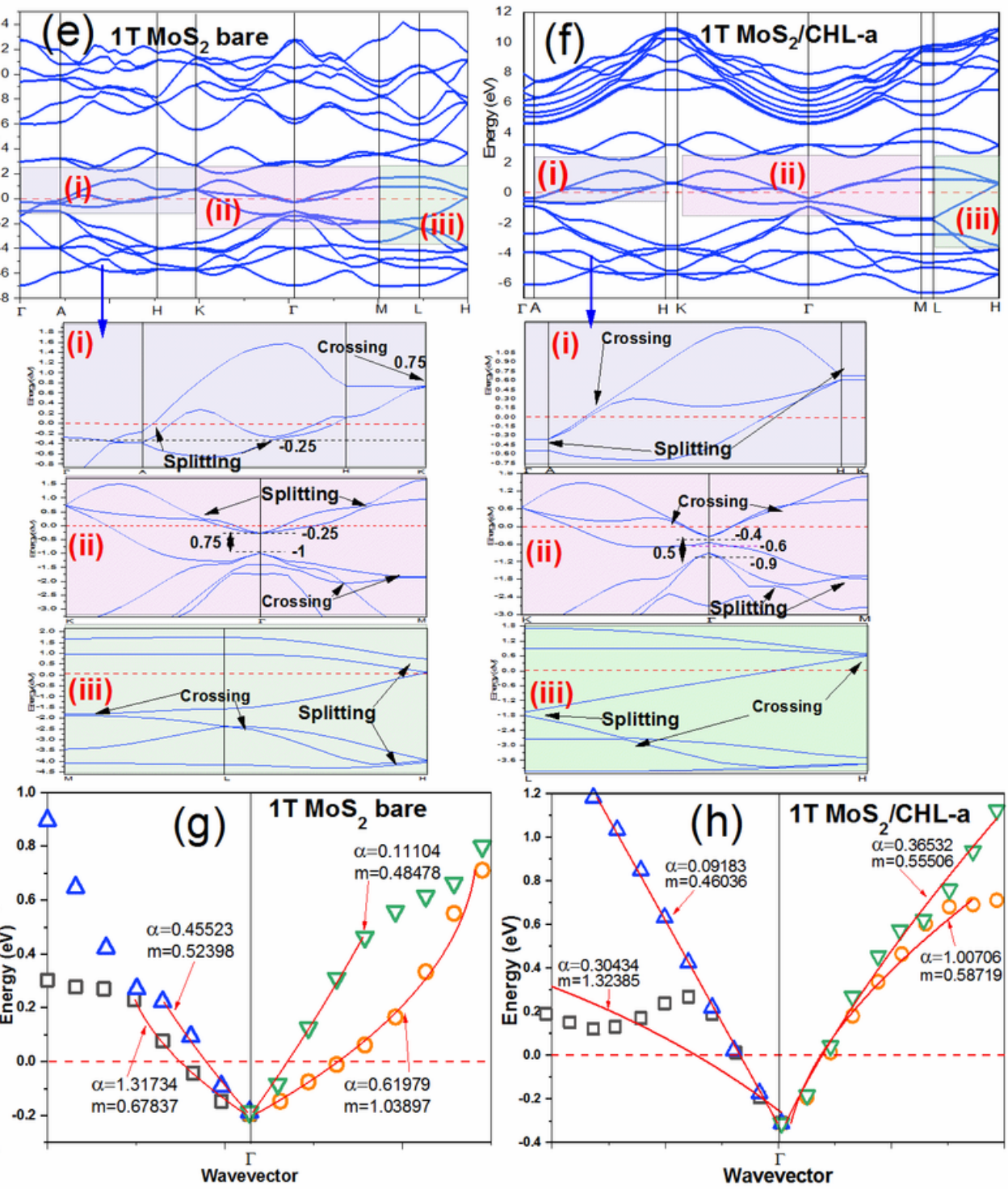

\section{Figure 3}

(a)Temperature-dependent IV spectra of chlorophyll MOS2, showing NDR effect in high-temperature range. (b) collapse diagram of Luttinger fitting. (c) "O: bias PDOS extracted from DFT showing the finite contribution of each of the s3p and $4 \mathrm{~d}$ orbital of $1 \mathrm{~T} \mathrm{MoS} 2 / \mathrm{CHL}-\mathrm{a}$ (c) in contradiction to bare counterpart(d).Detailed DFT features of bare 1T MoS2 and 1T MoS2/CHL-a (e),(f).The anisotropic dispersion of band on either direction of $\Gamma$ point fitted with a hyperbolic equation to extract the anisotropy of effective mass distribution which is greater in $1 \mathrm{~T} \mathrm{MoS} 2 / \mathrm{CHL}-\mathrm{a} .(\mathrm{g}) \&(\mathrm{~h})$ 

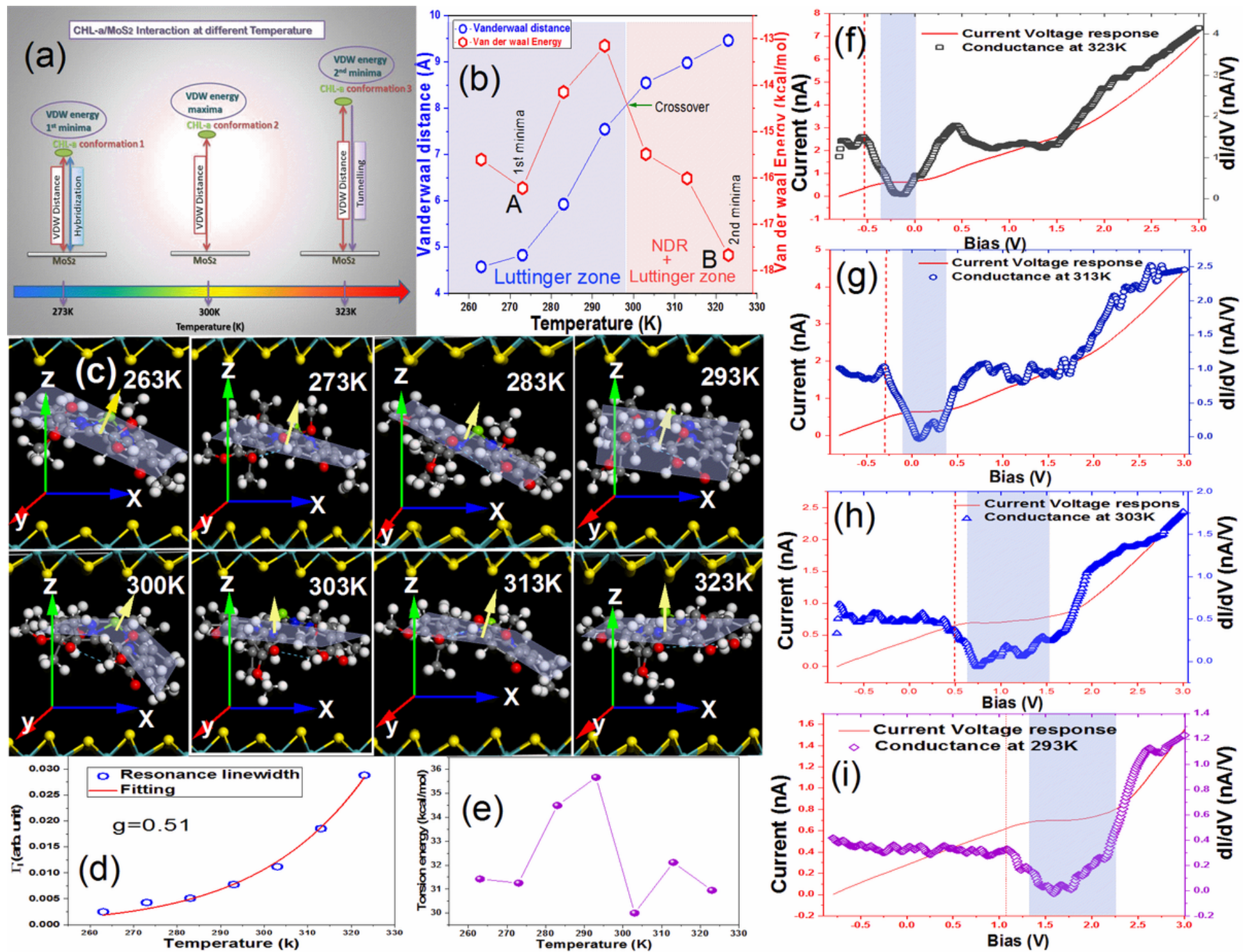

Figure 4

(a) overall schematic of MoS2 and CHL-a interaction in different temperature showing the evolution of NDR related to change in conformation and van der waal distance with temperature, (b)representing the temperature-dependent change in Van der waals distance and energy showing a crossover temperature dividing Luttinger and NDR region (c) temperature-dependent change in molecular geometry showing the change in molecular z-axis orientation to XY plane of CHL-a with 1T MoS2/CHL-a heterostructure, as obtained from MD simulation. (d)transmission probability obtained from Breit-Wigner line shape fitting (e)change in torsionenergy with temperature. ( $\mathrm{f}$ )-(i) temperature (323K to 293K) dependence conductance corresponding to various molecular geometry of $\mathrm{CHL}$-a with $1 \mathrm{~T} \mathrm{MoS} 2 / \mathrm{CHL}-a$ heterostructure.

\section{Supplementary Files}

This is a list of supplementary files associated with this preprint. Click to download.

- NSRsuppl.docx 